\title{
Generalized blind spots for dark matter direct detection in the 2HDM
}

\author{
M.E. Cabrera, ${ }^{a}$ J.A. Casas, ${ }^{b, c}$ A. Delgado ${ }^{c}$ and S. Robles ${ }^{d}$ \\ ${ }^{a}$ Instituto de Investigación en Ciencias Físicas y Matemáticas, ICFM-USAC, \\ Universidad de San Carlos de Guatemala, Ciudad de Guatemala, Guatemala \\ ${ }^{b}$ Instituto de Fúsica Teórica, IFT-UAM/CSIC, \\ Universidad Autónoma de Madrid, Cantoblanco, 28049 Madrid, Spain \\ ${ }^{c}$ Department of Physics, University of Notre Dame, \\ Notre Dame, IN 46556, U.S.A. \\ ${ }^{d} A R C$ Centre of Excellence for Particle Physics at the Terascale, \\ School of Physics, The University of Melbourne, Victoria 3010, Australia \\ E-mail: meugenia@ecfm.usac.edu.gt, j.alberto.casas@gmail.com, \\ antonio.delgado@nd.edu, sandra.robles@unimelb.edu.au
}

ABSTRACT: In this paper we study the presence of generalized blind spots, i.e. regions of the parameter space where the spin-independent cross section for dark matter direct detection is suppressed, in the context of a generic $2 \mathrm{HDM}$ and a minimal fermionic Higgs-portal dark sector. To this end, we derive analytical expressions for the couplings of the dark matter to the light and heavy Higgses, and thus for the blind spot solutions. Unlike the case of a standard Higgs sector, blind spots can occur even without a cancellation between different contributions, while keeping unsuppressed and efficient the annihilation processes in the early Universe involving Higgs states. As a consequence, the allowed parameter space is dramatically enhanced.

Keywords: Cosmology of Theories beyond the SM, Beyond Standard Model

ARXiv EPrint: 1912.01758 


\section{Contents}

1 Introduction 1

2 The model 2

3 Analytical expressions for DM couplings and blind spots 3

4 Blind spots in the alignment limit 5

4.1 Alignment from decoupling 6

4.2 Alignment without decoupling. Blind spots without cancellations 8

5 Summary and conclusions 12

\section{Introduction}

Models featuring stable weakly interacting massive particles (WIMPs) are still among the most popular explanations for dark matter (DM) [1]. However, the most appealing scenarios of this kind are under strong pressure, especially from direct detection (DD) experiments. More precisely, if the required annihilation of WIMP particles in the early Universe occurs through interactions with either the $Z$ or Higgs bosons (the so-called $Z$ - and Higgs-portals), which somehow represent the minimal DM-SM interactions, a large portion of the parameter space of the simplest models is already excluded (see e.g. refs. [2-9]). On the other hand, WIMP scenarios are still very attractive, not only for their simplicity, but also because they naturally arise in well-motivated beyond-the-SM (BSM) physics models. Popular way-outs to this experimental pressure occur when the annihilation in the early Universe is enhanced, as it happens when it takes place in a resonant form ("funnels") or through co-annihilation. These corners of the parameter space have attracted much attention, since they allow to save many interesting models of new physics.

There is another kind of regions of the parameter space where these economical WIMP scenarios can survive, namely when the DD elastic cross section is suppressed by some kind of cancellation. These are the so-called blind spots, which from the point of view of DM phenomenology offer a case of similar interest to the above mentioned funnel and co-annihilation regions. Blind spots have been examined in the context of specific supersymmetric models [10-13], and in simplified (scalar or fermion) singlet-doublet DM models, i.e. when the dark sector contains a singlet and a doublet which can mix up [14-17]. The latter case is specially interesting, as it represents the relevant DM sector of many scenarios, such as generalized supersymmetric models and extra-dimensions. 
On the other hand, a usual feature of many BSM frameworks is the presence of an extended Higgs sector, typically containing two (or more) Higgs doublets. Actually, twoHiggs-doublet models (2HDM) have received much attention on their own for assorted physical reasons, see e.g. refs. $[18,19]$. Thus, it is natural to wonder how the appearance of blind spots changes when the Higgs sector is a $2 \mathrm{HDM}$. The primary purpose of this paper is precisely to study this kind of scenario, deriving analytical expressions for the corresponding effective interactions of the WIMPs with ordinary matter, and thus for the blind-spot solutions. This will allow us to understand the general anatomy of the blind-spot regions for the different types of $2 \mathrm{HDMs}$. As we will see, the presence of the second Higgs enhances the size of the blind spots not only because there are new ways to obtain a cancellation of the spin-independent DD cross section (as already noticed in refs. [11, 15, 17]). Even without cancellations, the DD cross section can be small while keeping efficient the DM annihilation processes in the early Universe involving Higgs states. This leads to a dramatic enhancement of the viable regions of the parameter space.

The rest of the paper is summarized as follow. In section 2 we introduce the model. In section 3 we provide analytical expressions for the different DM couplings, and thus for the generalized blind-spot condition. Section 4 is devoted to the appearance of the blind-spot regions in the alignment limit. Finally, our conclusions are presented in section 5.

\section{The model}

As mentioned in the introduction, we will focus on a model with two Higgs doublets, $\Phi_{1}$, $\Phi_{2}$, with hypercharges $Y=1 / 2$ (our notation here follows that of ref. [20]). The dark sector consists of two fermionic $\mathrm{SU}(2)$ doublets $D_{1}, D_{2}$ ( "Higgsinos"), with hypercharges $+1 / 2$, $-1 / 2$ respectively, and a fermionic singlet $S$ ( "Singlino") with zero hypercharge. The $D_{1}, D_{2}$ states can be combined in a Dirac fermion, if desired. The notation here follows that of ref. [16], in order to facilitate comparisons. Note that this Dirac fermion represents the minimal UV completion of a fermion-singlet Higgs-portal scenario for DM.

The relevant terms of the most general Lagrangian for the dark sector are:

$$
-\mathcal{L} \supset \frac{1}{2} M_{S} S S+M_{D} D_{1} D_{2}+y_{1}^{1} S D_{1} \bar{\Phi}_{1}+y_{2}^{1} S D_{2} \Phi_{1}+y_{1}^{2} S D_{1} \bar{\Phi}_{2}+y_{2}^{2} S D_{2} \Phi_{2}+\text { h.c. },
$$

where $\bar{\Phi}_{1,2}=i \sigma_{2} \Phi_{1,2}^{*}$ and $M_{D}, M_{S}\left(y_{i}^{j}\right)$ are mass (Yukawa-coupling) parameters. The Higgs doublets acquire vacuum expectation values (VEVs) according to the structure of the 2HDM Higgs potential, which we do not write here explicitly (for further details see ref. [20]). Then, the CP-even neutral part of the Higgses reads

$$
\Phi_{1,2}=\frac{1}{\sqrt{2}}\left(\begin{array}{c}
0 \\
v_{1,2}+h_{1,2}^{0}
\end{array}\right), \bar{\Phi}_{1,2}=\frac{1}{\sqrt{2}}\left(\begin{array}{c}
v_{1,2}+h_{1,2}^{0} \\
0
\end{array}\right),
$$

where $v_{1}^{2}+v_{2}^{2}=v^{2}=(246 \mathrm{GeV})^{2}$. As usual, we define the $\tan \beta$ parameter so that

$$
v_{1}=v \cos \beta, \quad v_{2}=v \sin \beta .
$$


With these definitions, the relevant Lagrangian for the neutral states reads

$$
\begin{aligned}
-\mathcal{L} \supset & \frac{1}{2} M_{S} S S-M_{D} D_{1}^{0} D_{2}^{0}-\frac{1}{\sqrt{2}} y_{1}^{1} S D_{1}^{0}\left(v_{1}+h_{1}^{0}\right)+\frac{1}{\sqrt{2}} y_{2}^{1} S D_{2}^{0}\left(v_{1}+h_{1}^{0}\right) \\
& -\frac{1}{\sqrt{2}} y_{1}^{2} S D_{1}^{0}\left(v_{2}+h_{2}^{0}\right)+\frac{1}{\sqrt{2}} y_{2}^{2} S D_{2}^{0}\left(v_{2}+h_{2}^{0}\right)+\text { h.c. }
\end{aligned}
$$

Although in principle all the masses and couplings in the Lagrangian are complex, there are only three independent phases, namely those of $M_{S}^{*} M_{D}^{*} y_{1}^{1} y_{2}^{1}, y_{1}^{1}\left(y_{1}^{2}\right)^{*}, y_{2}^{1}\left(y_{2}^{2}\right)^{*}$, which we will assume to be real to avoid CP violations. This allows to take the six parameters of the Lagrangian as real quantities and to fix the sign of three of them. We will make use of this freedom later.

Of course, the fields appearing in the previous expression do not correspond to the mass eigenstates. For the Higgs sector the latter are $h^{0}, H^{0}$, i.e. the light (standard) and the heavy Higgses, respectively, which are related to the original $h_{1}^{0}, h_{2}^{0}$ fields by a basis rotation

$$
\left(\begin{array}{c}
h^{0} \\
H^{0}
\end{array}\right)=\left(\begin{array}{cc}
\cos \alpha & -\sin \alpha \\
\sin \alpha & \cos \alpha
\end{array}\right)\left(\begin{array}{l}
h_{2}^{0} \\
h_{1}^{0}
\end{array}\right) .
$$

The masses of the two physical Higgses $\left(m_{h^{0}}=125 \mathrm{GeV}, m_{H^{0}}\right)$ are determined by the structure of the Higgs potential. This is also true for the $\alpha$ angle, which, in principle, is independent of $\beta$; although in the decoupling limit $\left(m_{H^{0}} \rightarrow \infty\right)$ they are related by $\alpha=\beta-\pi / 2$.

Concerning the (neutral) fermionic sector, the "neutralino" mass eigenstates, $\chi_{1,2,3}^{0}$ arise upon diagonalization of the mass matrix $\mathcal{M}_{N}$, defined as

$$
-\mathcal{L}_{\text {mass }}=\frac{1}{2}\left(S, D_{1}^{0}, D_{2}^{0}\right) \mathcal{M}_{N}\left(\begin{array}{c}
S \\
D_{1}^{0} \\
D_{2}^{0}
\end{array}\right)+\text { h.c. },
$$

where, from eq. (2.4),

$$
\mathcal{M}_{N}=\left(\begin{array}{ccc}
M_{S} & -\frac{1}{\sqrt{2}}\left(y_{1}^{1} v_{1}+y_{1}^{2} v_{2}\right) & \frac{1}{\sqrt{2}}\left(y_{2}^{1} v_{1}+y_{2}^{2} v_{2}\right) \\
-\frac{1}{\sqrt{2}}\left(y_{1}^{1} v_{1}+y_{1}^{2} v_{2}\right) & 0 & -M_{D} \\
\frac{1}{\sqrt{2}}\left(y_{2}^{1} v_{1}+y_{2}^{2} v_{2}\right) & -M_{D} & 0
\end{array}\right) .
$$

Taking appropriate limits on the various parameters, we can recover simpler scenarios. In particular the relevant dark sector is a pure "Singlino" ("Higgsino") for $M_{D} \rightarrow \infty$ $\left(M_{S} \rightarrow \infty\right)$. Similarly, the scenario of just one (SM) Higgs is recovered for $m_{H^{0}} \rightarrow \infty$, $\alpha=\pi / 2-\beta$, as mentioned above.

\section{Analytical expressions for DM couplings and blind spots}

We are interested in the effective couplings, $y_{h \chi_{i} \chi_{i}}, y_{H \chi_{i} \chi_{i}}$ of the fermion mass eigenstates, $\chi_{i}^{0}$ (in particular $\chi_{1}^{0}$, which is the DM particle) to the physical Higgs states, $h^{0}, H^{0}$, i.e.

$$
-\mathcal{L} \supset y_{h \chi_{i} \chi_{i}} h^{0} \chi_{i}^{0} \chi_{i}^{0}+y_{H \chi_{i} \chi_{i}} H^{0} \chi_{i}^{0} \chi_{i}^{0} .
$$


Using eq. (2.5), these can be written in terms of the analogous couplings in the initial basis, namely

$$
\begin{aligned}
y_{h \chi_{i} \chi_{i}} & =-s_{\alpha} y_{h_{1} \chi_{i} \chi_{i}}+c_{\alpha} y_{h_{2} \chi_{i} \chi_{i}}, \\
y_{H \chi_{i} \chi_{i}} & =c_{\alpha} y_{h_{1} \chi_{i} \chi_{i}}+s_{\alpha} y_{h_{2} \chi_{i} \chi_{i}},
\end{aligned}
$$

where we have used the shorthand $s_{\phi}=\sin \phi, c_{\phi}=\cos \phi$. Now, from eqs. (2.4), (2.7), the $y_{h_{1,2} \chi_{i} \chi_{i}}$ couplings can be written as

$$
y_{h_{a} \chi_{i} \chi_{i}}= \pm \frac{1}{2} \frac{\partial m_{\chi_{i}^{0}}}{\partial v_{a}}, \quad a=1,2
$$

where the \pm sign corresponds to the case where the $m_{\chi_{i}}$ eigenvalue of the mass matrix is positive or negative. On the other hand, an analytical expression for $\partial m_{\chi_{i}^{0}} / \partial v_{a}$, and thus for $y_{h_{a} \chi_{i} \chi_{i}}$, can be obtained from the eigenvalue equation,

$$
\frac{\partial}{\partial v_{a}}\left|\mathcal{M}_{N}-m_{\chi_{i}^{0}} I\right|=0
$$

More precisely,

$$
y_{h_{a} \chi_{i} \chi_{i}}=-\frac{1}{2} \frac{\mathcal{N}_{a}}{\mathcal{D}} ; \quad a=1,2
$$

with

$$
\begin{aligned}
\mathcal{N}_{a}= & \pm M_{D}\left[y_{1}^{a}\left(y_{2}^{1} v_{1}+y_{2}^{2} v_{2}\right)+y_{2}^{a}\left(y_{1}^{1} v_{1}+y_{1}^{2} v_{2}\right)\right] \\
& +\left|m_{\chi_{i}^{0}}\right|\left[y_{1}^{a}\left(y_{1}^{1} v_{1}+y_{1}^{2} v_{2}\right)+y_{2}^{a}\left(y_{2}^{1} v_{1}+y_{2}^{2} v_{2}\right)\right],
\end{aligned}
$$

and

$$
\mathcal{D}= \pm 2\left|m_{\chi_{i}^{0}}\right| M_{S}-3 m_{\chi_{i}^{0}}^{2}+\frac{1}{2}\left[\left(y_{1}^{1} v_{1}+y_{1}^{2} v_{2}\right)^{2}+\left(y_{2}^{1} v_{1}+y_{2}^{2} v_{2}\right)^{2}\right]+M_{D}^{2}
$$

Plugging eq. (3.6) into eqs. (3.2), (3.3), we obtain analytical expressions for the couplings of $h^{0}$ and $H^{0}$ to the mass eigenstates:

$$
\begin{aligned}
& y_{h \chi_{i} \chi_{i}}=-\frac{1}{2 \mathcal{D}}\left\{ \pm M_{D}[\right.\left.y_{1}\left(y_{2}^{1} v_{1}+y_{2}^{2} v_{2}\right)+y_{2}\left(y_{1}^{1} v_{1}+y_{1}^{2} v_{2}\right)\right] \\
&\left.+\left|m_{\chi_{i}^{0}}\right|\left[y_{1}\left(y_{1}^{1} v_{1}+y_{1}^{2} v_{2}\right)+y_{2}\left(y_{2}^{1} v_{1}+y_{2}^{2} v_{2}\right)\right]\right\}, \\
& y_{H \chi_{i} \chi_{i}}=-\frac{1}{2 \mathcal{D}}\left\{ \pm M_{D}[\right.\left.\tilde{y}_{1}\left(y_{2}^{1} v_{1}+y_{2}^{2} v_{2}\right)+\tilde{y}_{2}\left(y_{1}^{1} v_{1}+y_{1}^{2} v_{2}\right)\right] \\
&\left.+\left|m_{\chi_{i}^{0}}\right|\left[\tilde{y}_{1}\left(y_{1}^{1} v_{1}+y_{1}^{2} v_{2}\right)+\tilde{y}_{2}\left(y_{2}^{1} v_{1}+y_{2}^{2} v_{2}\right)\right]\right\},
\end{aligned}
$$

where we have defined

$$
\begin{array}{ll}
y_{1}=-y_{1}^{1} s_{\alpha}+y_{1}^{2} c_{\alpha}, & y_{2}=-y_{2}^{1} s_{\alpha}+y_{2}^{2} c_{\alpha} \\
\tilde{y}_{1}=y_{1}^{1} c_{\alpha}+y_{1}^{2} s_{\alpha}, & \tilde{y}_{2}=y_{2}^{1} c_{\alpha}+y_{2}^{2} s_{\alpha} .
\end{array}
$$


Note that $y_{H^{0} \chi_{i} \chi_{i}}$ can be obtained from $y_{h^{0} \chi_{i} \chi_{i}}$ by simply replacing $y_{1,2} \rightarrow \tilde{y}_{1,2}$. The reason is simply that $y_{1,2}, \tilde{y}_{1,2}$ are the couplings of $h^{0}, H^{0}$ to the initial $D_{1,2}$ doublets. Namely, from eqs. (2.4), (2.5),

$$
-\mathcal{L} \supset-\frac{1}{\sqrt{2}} y_{1} S D_{1}^{0} h^{0}+\frac{1}{\sqrt{2}} y_{2} S D_{2}^{0} h^{0}-\frac{1}{\sqrt{2}} \tilde{y}_{1} S D_{1}^{0} H^{0}+\frac{1}{\sqrt{2}} \tilde{y}_{2} S D_{2}^{0} H^{0}+\text { h.c. },
$$

with $y_{1,2}, \tilde{y}_{1,2}$ given by eq. (3.11).

From expressions (3.9)-(3.11), it is straightforward to obtain the blind spots, i.e. the region of parameters where the spin-independent DD cross section is suppressed. Generically, the amplitude for the DM-nucleon scattering, $\chi_{1}^{0} N \rightarrow \chi_{1}^{0} N$, mediated by a Higgs $\left(h^{0}\right.$ or $H^{0}$ in $t$-channel) is proportional to the effective coupling, $y_{\mathrm{DD}}^{\text {eff }} / m_{h}^{2}$, with

$$
y_{\mathrm{DD}}^{\mathrm{eff}} \equiv \sum_{q}\left[y_{h \chi_{1} \chi_{1}}+\frac{m_{h}^{2}}{m_{H}^{2}} C_{q} y_{H \chi_{1} \chi_{1}}\right] f_{q}^{N} .
$$

Here $q$ runs over the quarks in the nucleon, $N ; f_{q}^{N}$ (with $N=p, n$ ) are the hadronic matrix elements, determined either experimentally or by lattice QCD simulations and related to the mass fraction of $q$ within the nucleon; and $C_{q}$ is a numerical factor that gives the departure of the coupling of the quark $q$ to the heavy Higgs, $H^{0}$, from that of the SM Higgs, $h^{0}$. The $C_{q}$ factors depend on the type of the $2 \mathrm{HDM}$ considered (more details in the next section). Whenever $y_{\mathrm{DD}}^{\mathrm{eff}} \simeq 0$, we are in a blind spot region of the parameter space.

\section{Blind spots in the alignment limit}

Experimental constraints indicate that the $125 \mathrm{GeV}$ Higgs of a $2 \mathrm{HDM}$ cannot be very different from the SM Higgs [21-26]. We adopt the conservative approach that the light Higgs is $100 \%$ SM-like (a situation which is usually called "alignment"), which effectively means that the heavy Higgs does not obtain a VEV, i.e. it is inert. The analysis of this model when the heavy Higgs is allowed to have a VEV consistent with the present experimental data will be postponed for a forthcoming paper. Consequently, from now on we will concentrate on the alignment limit to illustrate the structure of the blind spots.

The exact alignment limit (with the light Higgs, $h^{0}$, playing the role of the SM Higgs boson) occurs for $c_{\beta-\alpha}=0$, i.e. $\alpha=\beta-\pi / 2$. Then, we can recast expressions (3.9), (3.10) as

$$
\begin{aligned}
y_{h \chi_{i} \chi_{i}} & =-\frac{1}{2 \mathcal{D}} y^{2} v\left( \pm M_{D} \sin 2 \theta+\left|m_{\chi_{i}^{0}}\right|\right), \\
y_{H \chi_{i} \chi_{i}} & =-\frac{1}{2 \mathcal{D}} y \tilde{y} v\left( \pm M_{D} \sin (\theta+\tilde{\theta})+\left|m_{\chi_{i}^{0}}\right| \cos (\theta-\tilde{\theta})\right),
\end{aligned}
$$

with

$$
\begin{array}{lll}
y^{2}=\left(y_{1}\right)^{2}+\left(y_{2}\right)^{2}, & c_{\theta}=\frac{y_{1}}{y}, & s_{\theta}=\frac{y_{2}}{y}, \\
\tilde{y}^{2}=\left(\tilde{y}_{1}\right)^{2}+\left(\tilde{y}_{2}\right)^{2}, & c_{\tilde{\theta}}=\frac{\tilde{y}_{1}}{\tilde{y}}, & s_{\tilde{\theta}}=\frac{\tilde{y}_{2}}{\tilde{y}} .
\end{array}
$$


The expression for $\mathcal{D}$, eq. (3.8), is also simplified

$$
\mathcal{D}= \pm 2\left|m_{\chi_{i}^{0}}\right| M_{S}-3 m_{\chi_{i}^{0}}^{2}+\frac{1}{2} y^{2} v^{2}+M_{D}^{2} .
$$

Note that $y_{h \chi_{i} \chi_{i}} \propto y^{2}$, while $y_{H \chi_{i} \chi_{i}} \propto y \tilde{y}$. This occurs because in the alignment limit the Higgs VEV comes entirely from the doublet associated with the light Higgs, $h^{0}$, which thus appears always in the $v+h^{0}$ combination. Then, due to $\mathrm{SU}(2)$ invariance both effective couplings involve a $v$-factor and thereby a $y$-Yukawa, see eq. (3.12). For the same reason, it is easy to show that in this limit the expression (4.1) for $y_{h \chi_{i} \chi_{i}}$ can be obtained from

$$
y_{h \chi_{i} \chi_{i}}= \pm \frac{1}{2} \frac{\partial m_{\chi_{i}^{0}}}{\partial v}
$$

In the following, we make use of the freedom to fix the sign of three parameters to take $M_{S}, y_{1}$ and $y_{2}$ as positive, while the signs of $M_{D}, \tilde{y}_{1}, \tilde{y}_{2}$ can be positive or negative. Consequently, $\theta \in[0, \pi / 2], \tilde{\theta} \in[0,2 \pi]$. This convention allows to scan the whole parameter space in a complete and non-redundant way; and it converges to the sign convention used in ref. [16] for the case of a single Higgs.

\subsection{Alignment from decoupling}

A somewhat trivial way to obtain alignment is through decoupling, i.e. when $m_{H^{0}} \gg m_{h^{0}}$ (for details see ref. [20]). Then, the contribution of the heavy Higgs to the DD cross section becomes negligible and the effective coupling $y_{\mathrm{DD}}^{\text {eff }}$ of eq. (3.13) reads

$$
y_{\mathrm{DD}}^{\mathrm{eff}} \propto y_{h \chi_{1} \chi_{1}} \propto \pm M_{D} \sin 2 \theta+\left|m_{\chi_{1}^{0}}\right|
$$

which agrees with the expression obtained in ref. [16] for just one Higgs, as expected. Note that in this limit a blind spot is only possible when $M_{D}<0$. The reason is the following. From eq. (4.5), the blind spot condition, $y_{h \chi_{1} \chi_{1}}=0$, implies that $m_{\chi_{1}^{0}}$ does not depend on $v$, and thus must be equal to one of the mass eigenvalues of the mass matrix, eq. (2.7), when $v=0$, i.e. $M_{S}, M_{D}$ or $-M_{D}$. However, since $\pm M_{D} \sin 2 \theta+\left|m_{\chi_{1}^{0}}\right|=0$, this can only be achieved (barring the $\sin 2 \theta=1$ case) if $m_{\chi_{1}^{0}}=M_{S}$ (and thus positive) and $M_{D}<0$. Note also that in the decoupling limit the existence of a blind spot requires $M_{S} \leq\left|M_{D}\right|$, barring the aforementioned case.

All this is illustrated in the scan of figure 1 which shows the physically viable region in the $m_{\chi_{1}^{0}}-m_{D}$ plane where $\Omega_{\chi_{1}^{0}} \leq \Omega_{\mathrm{DM}}^{\mathrm{obs}}$, fulfilling DD bounds from XENON1T [27, 28] and PICO-60 [29]. The two narrow and dense strips at $m_{\chi_{1}^{0}}= \pm M_{D}$ correspond to models where $\chi_{1}^{0}$ is either almost a pure doublet, i.e. a combination of the $D_{1}^{0}, D_{2}^{0}$ fields; or a welltempered mixture of $S$ and $D_{1}^{0}, D_{2}^{0}$ [30]. Comparison of the upper branch (where there is no blind spot and the dark matter is in a well-tempered regime) with the lower one shows the noticeable effect of the blind spot. The $Z$ - and $h$-funnel regions are also visible in the plot.

The results for $\Omega_{\chi_{1}^{0}}=\Omega_{\mathrm{DM}}^{\mathrm{obs}}$ are very similar, except for the upper strip. The reason is that when $\chi_{1}^{0}$ is a pure doublet, the annihilation is "too efficient" except around $1 \mathrm{TeV}$, 


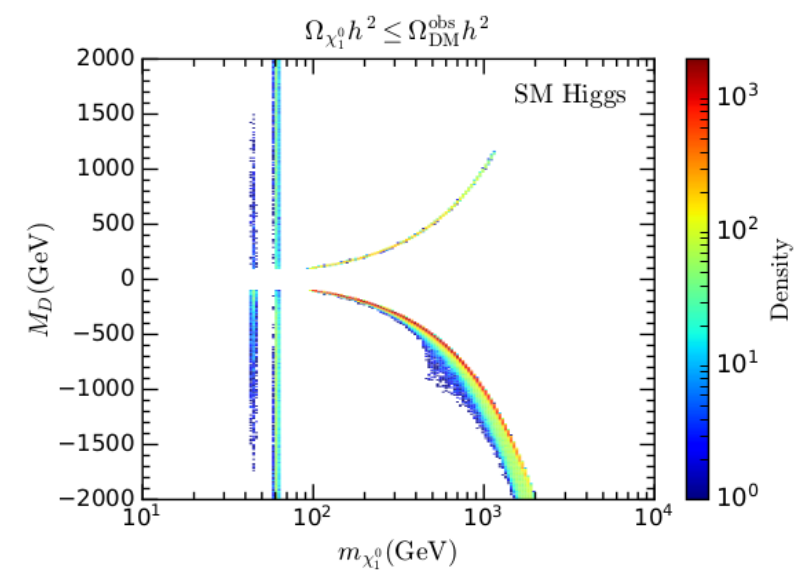

Figure 1. Regions of the parameter space allowing $\Omega_{\chi_{1}^{0}} h^{2} \leq \Omega_{\mathrm{DM}}^{\mathrm{obs}} h^{2}$ in the decoupling limit (equivalent to a SM Higgs sector). All the plotted points fulfill the bounds from XENON1T [27, 28] and PICO-60 [29]. Moreover the condition $\left|M_{D}\right|>100 \mathrm{GeV}$ has been required to satisfy the LEP limits [31] on charged fermions, in this case the charged components of the $D$-fields. The color code indicates the relative density of points.

which is the only piece of the upper strips that survives. On the other hand, in the welltempered regime it is possible to obtain the correct relic density with $M_{S} \sim\left|M_{D}\right| \sim m_{\chi_{1}^{0}}$, but at the price of raising the $y$-couplings in a way that DD excludes the model when $M_{D}>0$. In contrast, when $M_{D}<0$ the $y$-couplings can be arranged according to the blind spot relation, eq. (4.6) $\simeq 0$, thus evading DD bounds. Hence the lower strip survives in this regime, albeit not as a particularly dense region. Consequently, apart from the funnels and a narrow region at $\left|M_{D}\right| \simeq 1 \mathrm{TeV}$, all the regions rescued for $\Omega_{\chi_{1}^{0}}=\Omega_{\mathrm{DM}}^{\mathrm{obs}}$ correspond to the blind spot condition.

To perform the previous scan and those of the next subsection, we have implemented the model in FeynRules [32, 33], interfaced with CalcHEP [34]. More specifically, we have extended the publicly available $2 \mathrm{HDM}$ model files [35], considering only tree level interactions, with a singlet fermion and two doublet fermions as described in section 2. Then, the relic abundance and the elastic scattering cross sections have been calculated with microOMEGAS [36]. The scan has been performed in the following ranges of the relevant parameters:

$$
M_{S} \in[10,2000] \mathrm{GeV}, \quad M_{D} \in[-2000,2000] \mathrm{GeV}, \quad y_{1}, y_{2} \in[0,1],
$$

with a log prior on $M_{S}$ and flat priors on the remaining parameters, using MultiNest for an efficient exploration of the parameter space [37-39]. To that end, we have constructed a joint likelihood function, as follows

$$
\log \mathcal{L}_{\text {Joint }}=\log \mathcal{L}_{\Omega_{\mathrm{DM}} h^{2}}+\log \mathcal{L}_{\text {Xenon1T }},
$$

where $\mathcal{L}_{\Omega_{\mathrm{DM}} h^{2}}$ is implemented as an upper bound with a smeared step-function [40], centered at the observed value [41]. $\mathcal{L}_{\text {Xenon1T }}$ is calculated using RAPIDD [42], a surrogate model for fast computation of the expected DM spectrum in direct detection experiments, 


\begin{tabular}{|c|c|c|c|c|}
\hline & \multicolumn{2}{|c|}{ Type I } & \multicolumn{2}{c|}{ Type II } \\
\hline Higgs & $u$-quarks & $d$-quarks and leptons & $u$-quarks & $d$-quarks and leptons \\
\hline$h^{0}$ & $\cos \alpha / \sin \beta$ & $\cos \alpha / \sin \beta$ & $\cos \alpha / \sin \beta$ & $-\sin \alpha / \cos \beta$ \\
$H^{0}$ & $\sin \alpha / \sin \beta$ & $\sin \alpha / \sin \beta$ & $\sin \alpha / \sin \beta$ & $\cos \alpha / \cos \beta$ \\
\hline
\end{tabular}

Table 1. Factors for couplings of Higgs states to SM fermions in the Type I and Type II 2HDMs relative to those of the SM.

tuned to the latest XENON1T results $[27,28]$. Here and throughout the paper the DD cross section has been weighted by the $\xi=\min \left[1, \Omega_{\chi_{1}^{0}} / \Omega_{\mathrm{DM}}^{\mathrm{obs}}\right]$ factor, which appropriately scales the cross section for under-abundant dark matter.

It is apparent from figure 1 that, apart from the funnels, the absolute value of $M_{D}$ cannot be very different from $M_{S}$, even in the blind spot region. The reason is the following. Since in the decoupling limit the blind spot condition is equivalent to $y_{h \chi_{1} \chi_{1}}=0$, the coupling of the DM particle, $\chi_{1}^{0}$ to the SM Higgs vanishes for both cross sections DMnucleon elastic scattering and DM annihilation in the early Universe. In particular, the processes $\chi_{1}^{0} \chi_{1}^{0} \rightarrow h^{0} \rightarrow \mathrm{SM} \mathrm{SM}, \chi_{1}^{0} \chi_{1}^{0} \rightarrow h^{0} h^{0}$ vanish at first order. Consequently, in the blind spot the required annihilation occurs thanks to the $D$-component of $\chi_{1}^{0}$ and the corresponding weak interaction, as in the well-tempered regime. Since this component is inversely proportional to the difference of masses, if $\left|M_{D}\right|$ is much larger than $M_{S}$, the effective weak coupling of $\chi_{1}^{0}$ becomes too small to provide the required amount of DM annihilation. The results of this subsection are consistent with those obtained in ref. [16].

\subsection{Alignment without decoupling. Blind spots without cancellations}

A more interesting case arises when the alignment is achieved without decoupling [20, 43]. This occurs whenever the coupling in the Higgs-potential denoted by $Z_{6}$ in ref. [20] is vanishing or very small. Then, still $\alpha=\beta-\pi / 2$, and $m_{H^{0}}$ can be quite low without conflicting with any experimental constraints. The precise lower bound depends on the type of 2HDM under consideration [20].

In the Type I $2 \mathrm{HDM}$, defined by the fact that one of the initial doublets, say $\Phi_{2}$, is the only one that couples to all fermions, the bounds are very mild. Actually, $m_{H^{0}}$ can be close to $m_{h^{0}}=125 \mathrm{GeV}$ without conflicting with experiments. In the Type II 2HDM, in which $\Phi_{1}$ couples to down-like quarks and charged leptons, and $\Phi_{2}$ to up-like quarks, just as in supersymmetry, the bounds are more restrictive. This is mainly due to the limits from $H^{0} \rightarrow \tau^{+} \tau^{-}$, since in the Type II the coupling of $H^{0}$ to charged leptons is enhanced by $\tan \beta$ (see below). Generically, taking $m_{H^{0}} \geq 400 \mathrm{GeV}$ is safe, although it can be much lower (even below $200 \mathrm{GeV}$ ) if $m_{A} \geq 400 \mathrm{GeV}$ [20].

For DD matters, the most important difference between the Type I and Type II 2HDMs concerns the couplings to quarks, which are given in table 1 [20]

In the alignment limit, $\alpha=\beta-\pi / 2$ and we recover the SM couplings for the conventional Higgs, $h^{0}$. However, the couplings of the heavy Higgs, $H^{0}$, to $u$ - and $d$-quarks 


\begin{tabular}{|c|c|c|c|c|c|}
\hline \multicolumn{3}{|c|}{ Type I } & \multicolumn{3}{c|}{ Type II } \\
\hline$m_{H^{0}}(\mathrm{GeV})$ & $\tan \beta$ & $m_{A}=m_{H^{ \pm}}(\mathrm{GeV})$ & $m_{H^{0}}(\mathrm{GeV})$ & $\tan \beta$ & $m_{A}=m_{H^{ \pm}}(\mathrm{GeV})$ \\
\hline 300 & 5 & 600 & 300 & 5 & 600 \\
300 & 30 & 600 & 300 & 30 & 600 \\
800 & 5 & 800 & 800 & 5 & 800 \\
\hline
\end{tabular}

Table 2. Benchmarks for the Type I and Type II 2HDMs.

acquire the following factors

$$
\begin{aligned}
& C_{u}=-\cot \beta, \quad C_{d}=-\cot \beta \quad \text { (Type I), } \\
& C_{u}=-\cot \beta, \quad C_{d}=\tan \beta \quad \text { (Type II). }
\end{aligned}
$$

These are the $C_{q}$ coefficients to plug in expression (3.13) for the blind spot condition. An important point is that, with two Higgs states in play, $y_{\mathrm{DD}}^{\mathrm{eff}}$ can vanish not because the couplings of the DM particle to the Higgses vanish (unique possibility when there was just one Higgs), but because their contributions cancel in eq. (3.13). Consequently the blind spot condition can be accomplished and, simultaneously, DM particles can efficiently annihilate in the early Universe thanks to sizeable interactions with both Higgses. This opens enormously the available parameter space; in particular it is not necessary anymore that $\left|M_{D}\right|$ is close to $M_{S}$, as happened for the case of a unique Higgs (barring funnels and the "pure Higgsino" region).

Actually, $y_{\mathrm{DD}}^{\mathrm{eff}}$ can be small not due to a cancellation between the various terms in eq. (3.13), but simply because all of them are small (i.e. with no need of tuning), and still the annihilation of DM involving Higgses can be efficient enough. To see this, note first that the $H^{0}$ contribution to the DD cross section, given by the second term of eq. (3.13), can be small not because the $\tilde{y}$-couplings are small but because the prefactor $\frac{m_{h}^{2}}{m_{H}^{2}} C_{q}$ is. This is the typical case for the Type I $2 \mathrm{HDM}$, since $C_{q}=-\cot \beta$ and $\tan \beta \gtrsim 1$ to avoid a non-perturbative top Yukawa coupling. Then, if the $\tilde{y}$-couplings are sizeable, the processes $\chi_{1}^{0} \chi_{1}^{0} \rightarrow H^{0} \rightarrow$ SM SM, $\chi_{1}^{0} \chi_{1}^{0} \rightarrow H^{0} H^{0}, \chi_{1}^{0} \chi_{1}^{0} \rightarrow Z H^{0}$ and others can be efficient enough to provide the required DM annihilation. More generically, even if the above prefactor is $\mathcal{O}(1)$, the $H^{0}$ contribution in eq. (3.13) can be small because the $y$-couplings (not the $\tilde{y}$-couplings) are small, as can be seen from the expression for $y_{H \chi_{1} \chi_{1}}$, eq. (4.2). In that case, processes like $\chi_{1}^{0} \chi_{1}^{0} \rightarrow H^{0} H^{0}$ (proportional to $\tilde{y}^{2}$ ) can be equally efficient.

The bottom line of the previous paragraph is that, due to the presence of the second Higgs, the couplings involved in DM annihilation are not necessarily those involved in DD. This only happens for certain annihilation processes, as $\chi_{1}^{0} \chi_{1}^{0} \rightarrow h^{0} h^{0}$. As a result, large "blind spot" regions that were unviable in the decoupling (or one-Higgs) limit are now rescued. Actually, they are blind spots only in the sense that $y_{\mathrm{DD}}^{\mathrm{eff}}$ is very small, but this does not necessarily imply a cancellation between contributions.

We illustrate these facts in figure 2 (Type I), figure 3 (Type II) and figure 4, which are analogous to figure 1 , but for the benchmark models defined in table 2. The only difference in the scan procedure is that now the $\tilde{y}_{1}, \tilde{y}_{2}$ couplings have been also surveyed, 

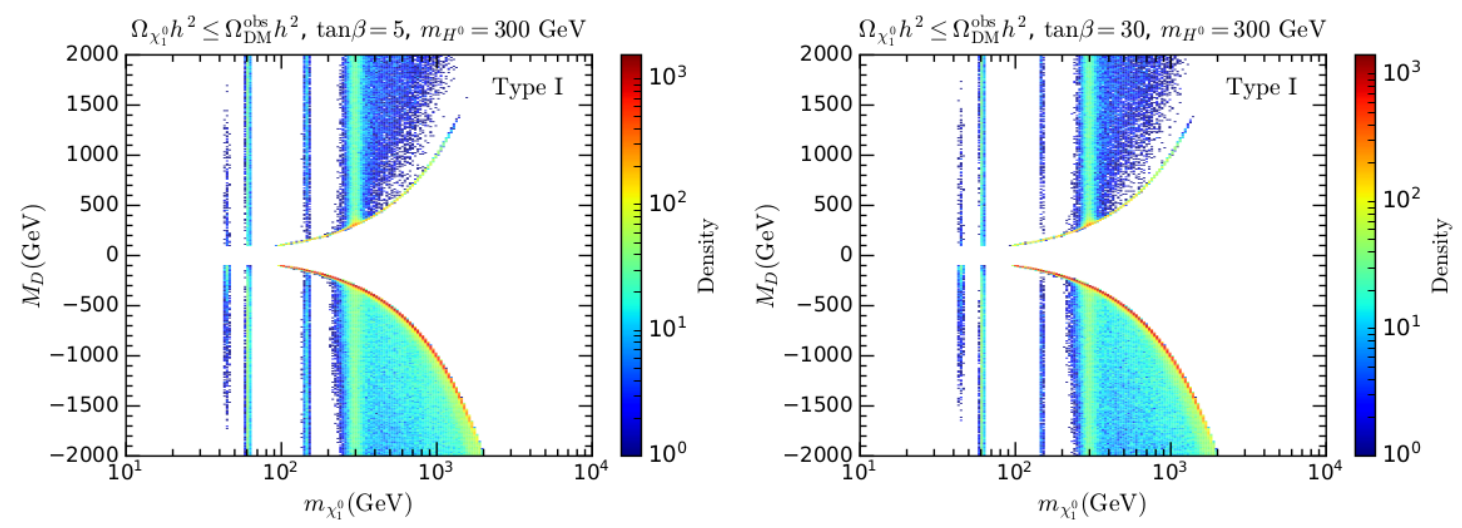

Figure 2. The same as figure 1, but for the Type I $2 \mathrm{HDM}$ with $\tan \beta=5$ (left panel) and $\tan \beta=30$ (right panel) with $m_{H^{0}}=300 \mathrm{GeV}$. The rest of the parameters are specified in table 2 .

similarly to $y_{1}, y_{2}$, but in the $[-1,1]$ range. The three figures show the dramatic enhancement of the regions of the parameter space consistent with the observed relic density and DD experiments, especially for $m_{\chi_{1}^{0}}$ above the $\chi_{1}^{0} \chi_{1}^{0} \rightarrow Z H^{0}$ threshold.

For all the benchmarks the values of $m_{H^{0}}, m_{A}, m_{H^{ \pm}}$have been chosen to be in the safe region with respect to experimental constraints [20]. It should be recalled here that their values, as well as that of $\tan \beta$, arise from the Higgs scalar potential, which we do not discuss here. For the purpose of this article they are free parameters. Interestingly, annihilation processes with $A, H^{ \pm}$in the final state can also be relevant for the relic density in some regions of the parameter space, even for the large masses considered here.

The aforementioned points can be clearly appreciated for the Type I 2HDM in figure 3. As in the case of a single Higgs in figure 1 , the region rescued for $\Omega_{\chi_{1}^{0}}=\Omega_{\mathrm{DM}}^{\mathrm{obs}}$ (not plotted) is very similar to that of $\Omega_{\chi_{1}^{0}} \leq \Omega_{\mathrm{DM}}^{\mathrm{obs}}$. Once more, the only difference between them are the narrow strips at $m_{\chi_{1}^{0}} \simeq \pm M_{D}$, which are not especially dense regions for $\Omega_{\chi_{1}^{0}}=\Omega_{\mathrm{DM}}^{\mathrm{obs}}$, except at the "pure Higgsino" solution, $M_{D} \simeq \pm 1 \mathrm{TeV}$. Aside from the various funnels visible in the plots at $m_{\chi_{1}^{0}} \simeq m_{Z}, m_{h^{0}}, m_{H^{0}}, \ldots$, all the allowed regions correspond to generalized blind spots, where $y_{\mathrm{DD}}^{\mathrm{eff}}$, as given by eq. (3.13), is nearly vanishing, though not necessarily by a cancellation between terms. As expected, the blind spot regions occur now for both positive and negative $M_{D}$, but interestingly there are still more solutions in the latter case. This is easily understood taking into account that in the Type I, the heavy Higgs contribution to $y_{\mathrm{DD}}^{\mathrm{eff}}$ is suppressed by the prefactor $\frac{m_{h}^{2}}{m_{H}^{2}} C_{q}=-\frac{m_{h}^{2}}{m_{H}^{2}} \cot \beta$. Then the light Higgs contribution, eq. (4.1), must be small as well, which can be more easily achieved for $M_{D}<0$, as discussed in the previous subsection. Let us also mention that the allowed regions are very similar for both $\tan \beta=5$ and $\tan \beta=30$.

The results for the Type II $2 \mathrm{HDM}$, given in figure 3 , are very similar. The only noticeable difference is that for $\tan \beta=30$ the allowed region is larger than for the other cases and, furthermore, it is almost identical for positive and negative $M_{D}$. The reason is the following. In the Type II, the prefactor of the heavy Higgs contribution to $y_{\mathrm{DD}}^{\mathrm{eff}}$ reads $\frac{m_{h}^{2}}{m_{H}^{2}} C_{q}=\frac{m_{h}^{2}}{m_{H}^{2}} \tan \beta$ for the $d$-quarks. For $\tan \beta=30$ this actually represents an 

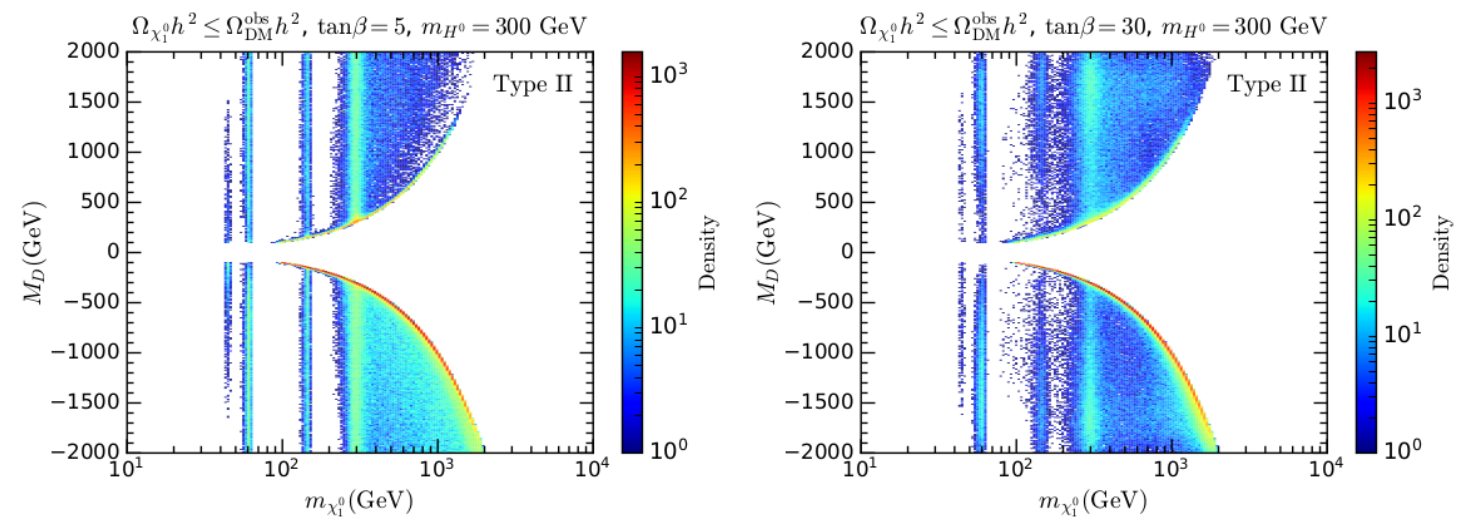

Figure 3. The same as figure 2 but for the Type II $2 \mathrm{HDM}$.
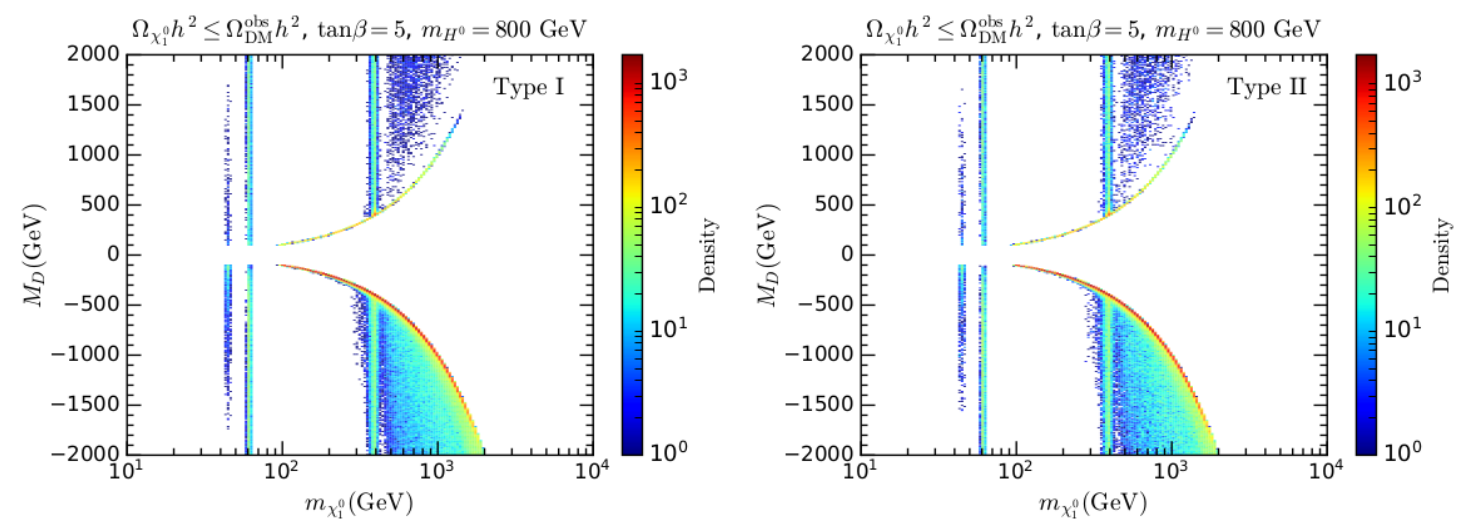

Figure 4. The same as figure 1 , but for the Type I $2 \mathrm{HDM}$ with $\tan \beta=5$ (left panel) and Type II $\tan \beta=5$ (right panel) with $m_{H^{0}}=800 \mathrm{GeV}$. The rest of the parameters are specified in table 2 .

enhancement, rather than a suppression. Hence, this term can be cancelled in eq. (3.13) with a sizeable light-Higgs contribution, and thus large $y$-couplings. In consequence, the processes $\chi_{1}^{0} \chi_{1}^{0} \rightarrow h^{0} \rightarrow$ SM SM, $\chi_{1}^{0} \chi_{1}^{0} \rightarrow h^{0} h^{0}$ can be now efficient for DM annihilation. This especially happens for $m_{\chi_{1}^{0}} \gtrsim 80 \mathrm{GeV}$, i.e. above the $W^{+} W^{-}$threshold. Likewise, since no small $y_{h \chi_{i} \chi_{i}}$ coupling is required now, the $M_{D}>0, M_{D}<0$ regions look alike.

The enhancement of the allowed regions holds even for rather large values of the extra Higgs states, especially above the mentioned $H^{0} Z$ threshold. This is illustrated in figure 4 for $m_{H^{0}}=m_{A}=m_{H^{ \pm}}=800 \mathrm{GeV}$ and $\tan \beta=5$.

We have seen that the DD cross section can be suppressed in the 2HDM by a variety of mechanisms, not necessarily a cancellation between terms. However, it is still true that, in order to obtain extremely suppressed DD cross sections some kind of cancellation for $y_{\mathrm{DD}}^{\mathrm{eff}}$ is required. Consequently, the density of viable models is higher when the DD cross section is not much smaller than the future experimental constraints, as illustrated in figure 5. This allows to be optimistic about the possibility that a scenario of the kind depicted in this paper might be detected by the next generation of direct detection experiments.

Finally, let us mention that there exist two additional 2HDMs, which are flavor chang- 


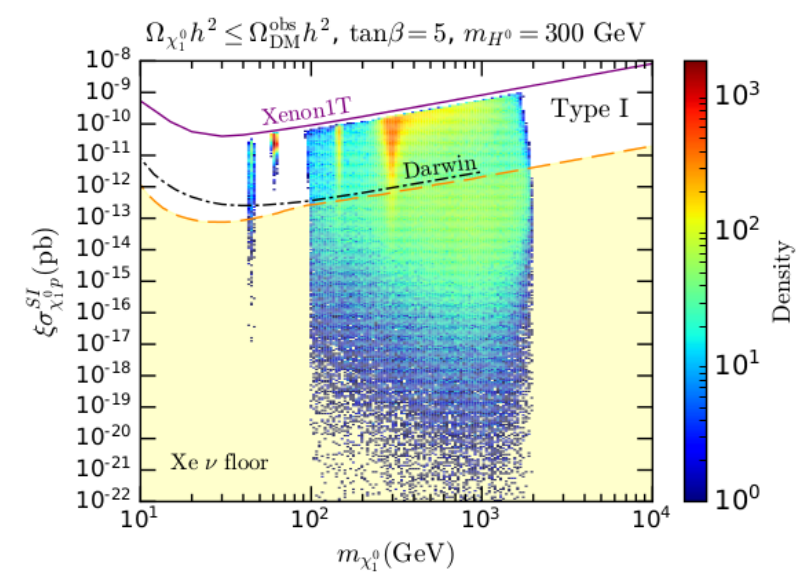

Figure 5. $\chi_{1}^{0}$-proton spin-independent elastic cross section, weighted by the scale factor $\xi$ for the Type I $2 \mathrm{HDM}$ benchmark with $\tan \beta=5$ and $m_{H^{0}}=300 \mathrm{GeV}$. The color code indicates the relative density of points. Upper-bound lines from XENON1T [27] and sensitivity projections from the future DARWIN experiment [44] are also shown, as well as the neutrino floor for a xenon target [45].

ing neutral current (FCNC) free: the so-called X (or "lepton-specific") and Y (or "flipped") models. The corresponding $C_{q}$ factors are the same as those of the Type I and Type II, respectively, so the results presented in figures 2,3 and 4 apply to them as well.

\section{Summary and conclusions}

$Z$ - and Higgs-portals are the most economical frameworks for WIMP dark matter. They are however under strong pressure (almost excluded), especially from direct detection experiments. One exception to this situation occurs when dark matter particles annihilate in a resonant way, i.e. the well-known $Z$-boson and Higgs funnels. Another way-out, equally interesting but not so explored, occurs when the spin-independent direct detection elastic cross section is suppressed due to some cancellation. These are the so-called "blind spots" of the parameter space. Both, funnels and blind spots, require some degree of tuning and, indeed, only rather narrow regions of the parameter space can be rescued in this way.

In this paper we have focused on the structure of the blind spots when the Higgs sector is not minimal, as it happens in many BSM scenarios; more precisely, we have assumed a generic 2HDM. In addition, we have considered a dark sector consisting of a neutral fermion plus a Dirac doublet. The latter represents the minimal UV completion of a fermion-singlet Higgs-portal scenario for dark matter. The funnel solutions change indeed little in this new framework, aside from the presence of additional funnels corresponding to the heavy Higgs and the pseudoscalar resonances. By contrast, the blind spot solutions change in a qualitative way, as discussed below.

In the first place, we have obtained general analytical expressions for the couplings of the dark matter to the light and heavy Higgses, which are the relevant ones for direct detection. This allowed us to write the effective coupling for direct detection, $y_{\mathrm{DD}}^{\text {eff }}$, and thus the explicit condition for a blind spot. In the case of a standard Higgs sector (which we 
re-visit as the decoupling limit of the $2 \mathrm{HDM}$ ) the vanishing of $y_{\mathrm{DD}}^{\mathrm{eff}}$ implies the suppression of annihilation processes in the early Universe involving the Higgs, $\chi_{1}^{0} \chi_{1}^{0} \rightarrow h^{0} \rightarrow \mathrm{SM}$ SM, $\chi_{1}^{0} \chi_{1}^{0} \rightarrow h^{0} h^{0}$. Then, dark matter annihilation can only occur thanks to a well-tempering mechanism, which implies an additional tuning. In particular, the masses of the singlet and the doublet must be rather close. In contrast, for a $2 \mathrm{HDM}, y_{\mathrm{DD}}^{\mathrm{eff}}$ can be small because there is a cancellation between the light and heavy Higgs contributions. Hence, the annihilation processes are not suppressed anymore, which enhances dramatically the allowed parameter space.

Actually, there is not even need of a cancellation between contributions. For example, for the Type I $2 \mathrm{HDM}$ the heavy Higgs contribution to $y_{\mathrm{DD}}^{\mathrm{eff}}$ is suppressed by its large mass, but also by an extra $\cot \beta$ factor. This means that the coupling of dark matter to the heavy Higgs can be large, making annihilation processes like $\chi_{1}^{0} \chi_{1}^{0} \rightarrow H^{0} H^{0}, \chi_{1}^{0} \chi_{1}^{0} \rightarrow Z H^{0}$ efficient, and still keeping direct detection cross sections suppressed.

We have illustrated these facts in the alignment (without decoupling) limit for the possible FCNC-free 2HDMs, using representative benchmarks. Interestingly, the enhancement of the allowed parameter space is very important even for large, $\mathcal{O}(1 \mathrm{TeV})$, masses of the extra Higgs states.

To summarize, the assumption of an extended Higgs sector has a great potential to rescue theoretically appealing WIMP scenarios.

\section{Acknowledgments}

The work of JAC has been partially supported by Spanish Agencia Estatal de Investigación through the grant "IFT Centro de Excelencia Severo Ochoa SEV-2016-0597" and by MINECO project FPA 2016-78022-P. The work of AD was partially supported by the National Science Foundation under grant PHY-1820860. SR was supported by the Australian Research Council. We thank Andrew Cheek for providing us with the RAPIDD tool tailored to XENON1T.

Open Access. This article is distributed under the terms of the Creative Commons Attribution License (CC-BY 4.0), which permits any use, distribution and reproduction in any medium, provided the original author(s) and source are credited.

\section{References}

[1] G. Bertone, D. Hooper and J. Silk, Particle dark matter: evidence, candidates and constraints, Phys. Rept. 405 (2005) 279 [hep-ph/0404175] [INSPIRE].

[2] G. Arcadi, Y. Mambrini and F. Richard, Z-portal dark matter, JCAP 03 (2015) 018 [arXiv: 1411.2985] [INSPIRE].

[3] A. Alves, A. Berlin, S. Profumo and F.S. Queiroz, Dark matter complementarity and the $Z^{\prime}$ portal, Phys. Rev. D 92 (2015) 083004 [arXiv:1501.03490] [INSPIRE].

[4] M. Escudero, A. Berlin, D. Hooper and M.-X. Lin, Toward (finally!) ruling out $Z$ and Higgs mediated dark matter models, JCAP 12 (2016) 029 [arXiv: 1609.09079] [INSPIRE]. 
[5] G. Arcadi et al., The waning of the WIMP? A review of models, searches and constraints, Eur. Phys. J. C 78 (2018) 203 [arXiv: 1703.07364] [InSPIRE].

[6] C. Gross, O. Lebedev and T. Toma, Cancellation mechanism for dark-matter-nucleon interaction, Phys. Rev. Lett. 119 (2017) 191801 [arXiv:1708.02253] [INSPIRE].

[7] J.A. Casas, D.G. Cerdeño, J.M. Moreno and J. Quilis, Reopening the Higgs portal for single scalar dark matter, JHEP 05 (2017) 036 [arXiv:1701.08134] [INSPIRE].

[8] S. Baum, M. Carena, N.R. Shah and C.E.M. Wagner, Higgs portals for thermal dark matter. EFT perspectives and the NMSSM, JHEP 04 (2018) 069 [arXiv: 1712.09873] [INSPIRE].

[9] G. Arcadi, A. Djouadi and M. Raidal, Dark matter through the Higgs portal, Phys. Rept. 842 (2020) 1 [arXiv: 1903.03616] [inSPIRE].

[10] C. Cheung, L.J. Hall, D. Pinner and J.T. Ruderman, Prospects and blind spots for neutralino dark matter, JHEP 05 (2013) 100 [arXiv:1211.4873] [INSPIRE].

[11] P. Huang and C.E.M. Wagner, Blind spots for neutralino dark matter in the MSSM with an intermediate $m_{A}$, Phys. Rev. D 90 (2014) 015018 [arXiv: 1404.0392] [INSPIRE].

[12] A. Anandakrishnan, B. Shakya and K. Sinha, Dark matter at the pseudoscalar Higgs resonance in the phenomenological MSSM and SUSY GUTs, Phys. Rev. D 91 (2015) 035029 [arXiv: 1410.0356] [INSPIRE].

[13] A. Crivellin, M. Hoferichter, M. Procura and L.C. Tunstall, Light stops, blind spots and isospin violation in the MSSM, JHEP 07 (2015) 129 [arXiv:1503.03478] [INSPIRE].

[14] C. Cheung and D. Sanford, Simplified models of mixed dark matter, JCAP 02 (2014) 011 [arXiv: 1311.5896] [INSPIRE].

[15] A. Berlin, S. Gori, T. Lin and L.-T. Wang, Pseudoscalar portal dark matter, Phys. Rev. D 92 (2015) 015005 [arXiv: 1502.06000] [INSPIRE].

[16] S. Banerjee, S. Matsumoto, K. Mukaida and Y.-L.S. Tsai, WIMP dark matter in a well-tempered regime: a case study on singlet-doublets fermionic WIMP, JHEP 11 (2016) 070 [arXiv: 1603. 07387] [INSPIRE].

[17] G. Arcadi, 2HDM portal for singlet-doublet dark matter, Eur. Phys. J. C 78 (2018) 864 [arXiv: 1804.04930] [INSPIRE].

[18] G.C. Branco et al., Theory and phenomenology of two-Higgs-doublet models, Phys. Rept. 516 (2012) 1 [arXiv:1106.0034] [INSPIRE].

[19] X.-M. Jiang et al., Pseudo-Nambu-Goldstone dark matter and two-Higgs-doublet models, Phys. Rev. D 100 (2019) 075011 [arXiv:1907.09684] [INSPIRE].

[20] J. Bernon et al., Scrutinizing the alignment limit in two-Higgs-doublet models: $m_{h}=125 \mathrm{GeV}$, Phys. Rev. D 92 (2015) 075004 [arXiv: 1507.00933] [INSPIRE].

[21] ATLAS collaboration, Observation of a new particle in the search for the standard model Higgs boson with the ATLAS detector at the LHC, Phys. Lett. B 716 (2012) 1 [arXiv: 1207.7214] [INSPIRE].

[22] CMS collaboration, Observation of a new boson at a mass of $125 \mathrm{GeV}$ with the CMS experiment at the LHC, Phys. Lett. B $\mathbf{7 1 6}$ (2012) 30 [arXiv:1207.7235] [INSPIRE].

[23] ATLAS collaboration, Measurements of Higgs boson production and couplings in diboson final states with the ATLAS detector at the LHC, Phys. Lett. B 726 (2013) 88 [Erratum ibid. B 734 (2014) 406] [arXiv: 1307.1427] [INSPIRE]. 
[24] CMS collaboration, Observation of a new boson with mass near $125 \mathrm{GeV}$ in pp collisions at $\sqrt{s}=7$ and $8 \mathrm{TeV}$, JHEP 06 (2013) 081 [arXiv:1303.4571] [INSPIRE].

[25] CMS collaboration, Measurements of properties of the Higgs boson decaying into the four-lepton final state in pp collisions at $\sqrt{s}=13$ TeV, JHEP 11 (2017) 047 [arXiv: 1706.09936] [INSPIRE].

[26] ATLAS collaboration, Measurement of the Higgs boson mass in the $H \rightarrow Z Z^{*} \rightarrow 4 \ell$ and $H \rightarrow \gamma \gamma$ channels with $\sqrt{s}=13$ TeV pp collisions using the ATLAS detector, Phys. Lett. B 784 (2018) 345 [arXiv: 1806.00242] [INSPIRE].

[27] XENON collaboration, Dark matter search results from a one ton-year exposure of XENON1T, Phys. Rev. Lett. 121 (2018) 111302 [arXiv:1805.12562] [INSPIRE].

[28] XENON collaboration, Constraining the spin-dependent WIMP-nucleon cross sections with XENON1T, Phys. Rev. Lett. 122 (2019) 141301 [arXiv:1902.03234] [INSPIRE].

[29] PICO collaboration, Dark matter search results from the complete exposure of the PICO-60 $C_{3} F_{8}$ bubble chamber, Phys. Rev. D 100 (2019) 022001 [arXiv:1902.04031] [INSPIRE].

[30] N. Arkani-Hamed, A. Delgado and G.F. Giudice, The well-tempered neutralino, Nucl. Phys. B 741 (2006) 108 [hep-ph/0601041] [INSPIRE].

[31] ALEPH collaboration, Absolute mass lower limit for the lightest neutralino of the MSSM from $e^{+} e^{-}$data at $\sqrt{s}$ up to $209 \mathrm{GeV}$, Phys. Lett. B 583 (2004) 247 [INSPIRE].

[32] N.D. Christensen and C. Duhr, FeynRules - Feynman rules made easy, Comput. Phys. Commun. 180 (2009) 1614 [arXiv:0806.4194] [INSPIRE].

[33] A. Alloul et al., FeynRules 2.0 - A complete toolbox for tree-level phenomenology, Comput. Phys. Commun. 185 (2014) 2250 [arXiv:1310.1921] [INSPIRE].

[34] N.D. Christensen et al., A comprehensive approach to new physics simulations, Eur. Phys. J. C 71 (2011) 1541 [arXiv:0906.2474] [INSPIRE].

[35] C. Degrande, Automatic evaluation of UV and R2 terms for beyond the standard model Lagrangians: a proof-of-principle, Comput. Phys. Commun. 197 (2015) 239 [arXiv: 1406.3030] [INSPIRE].

[36] G. Bélanger, F. Boudjema, A. Pukhov and A. Semenov, MicrOMEGAs 2.0: a program to calculate the relic density of dark matter in a generic model, Comput. Phys. Commun. 176 (2007) 367 [hep-ph/0607059] [INSPIRE].

[37] F. Feroz and M.P. Hobson, Multimodal nested sampling: an efficient and robust alternative to MCMC methods for astronomical data analysis, Mon. Not. Roy. Astron. Soc. 384 (2008) 449 [arXiv: 0704.3704].

[38] F. Feroz, M.P. Hobson and M. Bridges, MultiNest: an efficient and robust Bayesian inference tool for cosmology and particle physics, Mon. Not. Roy. Astron. Soc. 398 (2009) 1601 [arXiv:0809.3437].

[39] F. Feroz, M.P. Hobson, E. Cameron and A.N. Pettitt, Importance nested sampling and the MultiNest algorithm, arXiv:1306.2144 [INSPIRE].

[40] R. Ruiz de Austri, R. Trotta and L. Roszkowski, A Markov chain Monte Carlo analysis of the CMSSM, JHEP 05 (2006) 002 [hep-ph/0602028] [INSPIRE].

[41] Planck collaboration, Planck 2018 results. VI. Cosmological parameters, arXiv: 1807.06209 [INSPIRE]. 
[42] D.G. Cerdeño, A. Cheek, E. Reid and H. Schulz, Surrogate models for direct dark matter detection, JCAP 08 (2018) 011 [arXiv: 1802.03174] [INSPIRE].

[43] M. Carena, I. Low, N.R. Shah and C.E.M. Wagner, Impersonating the standard model Higgs boson: alignment without decoupling, JHEP 04 (2014) 015 [arXiv:1310.2248] [INSPIRE].

[44] DARWIN collaboration, DARWIN: towards the ultimate dark matter detector, JCAP 11 (2016) 017 [arXiv : 1606.07001] [INSPIRE].

[45] F. Ruppin, J. Billard, E. Figueroa-Feliciano and L. Strigari, Complementarity of dark matter detectors in light of the neutrino background, Phys. Rev. D 90 (2014) 083510 [arXiv: 1408.3581] [INSPIRE]. 